\title{
Prevention of medication errors: a lot to be done in Brazil!
}

\section{Prevenção de erros de medicação: muito a ser feito no Brasil!}

Globally, low quality health care is estimated to cause injury to about $10 \%$ of patients, and $15 \%$ of such harm is caused by medicines ${ }^{1}$. Adverse Drug Events (ADEs) are frequent incidents and are considered important cause of preventable harm in health systems around the world. The cost associated with the occurrence of preventable ADEs has been globally estimated at US\$ 42 billion a year ${ }^{2}$.

Looking at the importance of the topic, the World Health Organization (WHO) launched in 2017 the 3rd Global Challenge, "Medication without Harm"2, whose main focus is reducing severe avoidable medication-related harm. Most of the times, adverse drug reactions are unavoidable. The challenge's goal is to reduce the serious harm caused by ADEs by $50 \%$ in five years. This goal is focused on results (outcomes) and not in process indicators. In contrast, most of the $\mathrm{ADE}$ indicators used to assess medication safety in Brazil are process indicators. It is recommended to use outcome indicators and it should be added that health care interventions are best evaluated using outcome indicators whenever possible. There are already instrument proposals using outcome indicators, the "thermometer", used in England, but which needs to be tested in other environments and diverse conditions $s^{3}$.

In Brazil, with the creation of the National Patient Safety Program in 2013 (Brazilian Ministry of Healthy - Portaria 529/2013 and Brazilian Regulatory Agency - Anvisa - RDC 36/2013), all health facilities were required to implement six protocols from the Ministry of Health in addition to other activities. The protocol dealing with medication errors describes several effective interventions that can improve medication safety4.

Another important issue to be discussed is reporting of ADE. When we analyze the ADE reported to Anvisa a significant underreporting can be verified, apart from misreporting (Notivisa and Vigimed). It is possible to identify the main causes of these inadequacies: notifiers may not really know what ADEs are, they are not using active and effective methods for registration, and there is a sense of guilt and fear of punishment that prevents ADEs from being reported.

On the other hand, in the literature available in Brazil, the term adverse drug reactions is more often used, and little is mentioned about medication errors. It is necessary to better understand what ADEs are. The $\mathrm{WHO}$ proposes measures to improve the prevention of ADEs $s^{5}$. For the prevention of ADEs, it is necessary to implement measures aimed at four main objectives: patients, medicines as products, healthcare professionals, and medication systems and practices. Also, three priority areas should be focused on: high-risk situations (patients and medicines), polypharmacy and care transitions ${ }^{2}$.

The patient should be the main focus in the prevention of the ADEs. The pharmacist plays a key role in the inclusion and training of patients at all health care levels ${ }^{2}$. Partnering with the patient helps to add one last barrier of safety to avoid harm when the medicine reaches the patient.

The healthcare professional should be another focus where the working and environmental conditions, stress, fatigue, forms of continuing education and other situations should be considered. Training in ADEs should be modified for active methods, training in-services and simulations. Traditional education with formal classes is proving to be ineffective for changes in care. It is necessary to engage patients and professionals with methods that work at an emotional level to change commitment ${ }^{6}$.

Medicines as products still have serious problems in the Brazilian market with similar names and packaging and confusing labeling. Anvisa has been developing some activities, such as the revision of RDC $71 / 2009$, which deals with labels and packaging and the modification of visual identity (packaging all green) for the medications produced for the federal government. The green packaging has provoked errors and there are already working groups at Anvisa and the Brazilian Ministry of Health that have made proposals for changes. The realization of these changes is important for both the patients and the healthcare professionals.

It is relevant to mention that several pharmaceutical industries produce medicines ready for use, in unit doses and with bar codes, improving safety and avoiding rework in the pharmacy. The less we handle medicines, the safer the product. Why not giving preference to the acquisition of these medicines?

Access to the information on medications must be fast, reliable and constantly updated. The Federal Pharmacy Council, for example, provided pharmacists with free access to one of the best drug information databases in the world (Micromedex). Why do few pharmacists use this tool?

Technologies, especially automation, must be carefully implemented, such as robots, adoption of electronic prescription with clinical support, bar code, closed loop drug administration, automated medication

\section{Presidente do Instituto para Práticas Seguras no Uso de Medicamentos,} ISMP BRASIL

Submitted: 06/05/19

Accepted: $01 / 08 / 19$

DOI: $10.30968 /$ rbfhss.2019.103.0449 ISSN online: $2316-7750$ 
dispensing systems, and others. However, it is necessary to evaluate the new errors that these technologies might introduce. After implementation, there should be systems to monitor for unanticipated problems and to use appropriate indicators to check whether the new technologies actually led to improvements.

According to the $\mathrm{WHO}^{2}$, it is necessary to prioritize high-risk situations concerning patients and medicines to prevent ADEs. The patients should be considered at risk in the following situations: extremes of age, renal and liver failure, pregnant women, and patients with multiple diseases. Considering different hospital profiles, other patients may be classified as at risk.

As for the prioritization of medicines, some of them have greater potential to cause serious harm and death to patients when failures occur during their use. Due to this high potential for harm, antimicrobial agents; potassium chloride and other concentrated electrolytes; insulin; opioids and other sedatives; antineoplastic agents; and heparin and other anticoagulants have been defined as priority medications in the Global Challenge ${ }^{2}$. Most priority drugs are known as high alert medications (HAM). Depending on the type of hospital, other HAM can be prioritized.

With regard to drug therapy specifically, it is known that the transition of care, especially to and from hospital environments, significantly increases the occurrences of ADEs, mainly due to communication failures. To prevent ADEs and to ensure a safe transition, especially between different health care sites, the use of strategies that improve communication is encouraged. The implementation of medication reconciliation in this context is fundamental?

Meta-analyses and systematic reviews generally reinforce that the involvement of pharmacists in intensive care units, particularly in bedside rounds and pharmacotherapy review activities, reduces medication errors. ${ }^{8}$. This is an important aspect to be discussed because in many Brazilian hospitals there is shortage or an insufficient number of clinical pharmacists. It is still difficult to convince hospital leaders of the need to implement clinical pharmacy or to hire clinical pharmacists in sufficient numbers for the necessary activities. This difficulty is reinforced by the financial crisis that is devastating Brazil. Pharmacists should take actions to document objectively and in convincing ways the interventions that clinical pharmacy has taken to produce an indispensable positive cost/benefit relationship, along with improvements in quality/safety.

It is common to verify the use of interventions for the prevention of ADEs without knowing the baseline incidence of medication-related harm and which part of the medication use process failed. That is to say that, in this situation, it is not known where the weakest points are in the system and where the most urgent interventions for improvements are needed. Working without a “diagnosis" of the system's health is like working blind. Diagnoses need to be made, action plans drawn up and implemented, the entire system needs to be monitored, and the impact of the interventions evaluated with an appropriate methodology. The Institute for Safe Medications Practices (ISMP) Brazil has translated and adapted one of the best tools in the world for the evaluation of medication safety in hospitals. This questionnaire has already been applied in 21 Brazilian hospitals and is being adopted by another $11^{9}$. We suggest that the hospitals make a diagnosis before starting to implement interventions.

Finally, when the various causes of ADEs are analyzed, there are no simple solutions because these origins are often complex. The multifaceted measures are, in general, those that show the best results and the medication process must be simplified as possible. Important steps have already been taken in Brazil in the prevention of ADEs; however, much progress still needs to be made in order to have real medication safety at any level of care.

\section{References}

1. De Vries EN, Ramrattan MA, Smorenburg SM, et al. The incidence and nature of in-hospital adverse events: a systematic review. Qual Saf Health Care. 2008; $17: 216-23$.

2. Organização Mundial da Saúde. Medication Without Harm: WHO's Third Global Patient Safety Challenge. Genebra: OMS; 2017.

3. Ostami P, Power M, Harrison A, et al. Learning from the design, development and implementation of the Medication Safety Thermometer. International Journal for Quality in Health Care, 2017,29(2), 301-309.

4. Brasil. Ministério da Saúde, Anvisa, Fiocruz, Fhemig. Anexo 03 da Portaria MS no 2.095 (24.09.2013) - Protocolo de Segurança na Prescrição, uso e administração de medicamentos. 2013.

5. Organização Mundial da Saúde. WHO Global Patient Safety Challenge: Reporting and learning systems for medication errors: the role of pharmacovigilance centres. Genebra: Organização Mundial da Saúde; 2014. ISBN 978924150794 3.96p.

6. Heath C, Heath D. A guinada. São Paulo: Best Bussiness. ISBN: 8576843293.2010.294p.

7. Instituto para Práticas Seguras no Uso de Medicamentos. Prevenção de erros de medicação na transição do cuidado. Boletim ISMP Brasil. 2019 Mar; 8(2):1-11.

8. Shekelle PG, Wachter RM, Pronovost PJ, et al. Making health care safer II: an updated critical analysis of the evidence for patient safety practices. Rockville, MD: Agency for Healthcare Research and Quality; 2013 Mar. Report no.: 211.

9. Instituto para Práticas Seguras no Uso de Medicamentos. ISMP. Questionário de Autoavaliação sobre a Segurança do Sistema de Utilização de Medicamentos em Hospitais. Belo Horizonte: ISMP Brasil; 2015 [Internet]. Disponível em: http://questionario.ismp-brasil.org/QUESTIONARIO_ISMP_BRASIL.pdf. 